\title{
Qualidade de vida e fatores associados em idosos dependentes em uma cidade do interior do Nordeste
}

\author{
Quality of life and associated factors in functionally \\ dependent elderly in the hinterland of Northeast Brazil
}

Gilson de Vasconcelos Torres', Luciana Araújo dos Reis², Luana Araújo dos Reis ${ }^{3}$, Marcos Henrique Fernandes

\section{RESUMO}

Objetivo: Este estudo tem por objetivo verificar a influência de fatores sociodemográficos, condições de saúde, capacidade funcional e dinâmica familiar na qualidade de vida de idosos dependentes residentes em domićlío em uma cidade do interior da região do Nordeste.

Métodos: Trata-se de uma pesquisa de caráter analítico com delineamento transversal. A amostra deste estudo foi composta por 117 idosos dependentes, cadastrados nas Unidades de Saúde da Família da área de abrangência do bairro do Jequiezinho, no município de Jequié, BA. Os instrumentos de coleta de dados utilizados foram o WHOQOL-OLD, o Índice de Barthel, o Apgar de Família e o levantamento de dados sociodemográficos e condições

\section{Palavras-chave}

Idosos dependentes, condições de saúde, domićlio, dados sociais e demográficos, família e qualidade de vida.

\section{Keywords}

Dependent elderly, health status, domicile, social and demography data, family and quality of life.

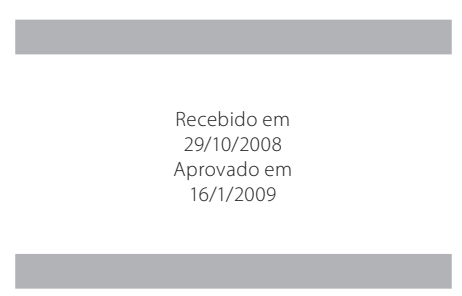

de saúde. Resultados: Com a aplicação do Teste do qui-quadrado $\left(x^{2}\right)$ encontrou-se diferença estatística entre comprometimento da qualidade de vida e da dinâmica familiar, com exceção do domínio autonomia ( $p=0,061)$ da qualidade de vida. Conclusões: Apenas o comprometimento da dinâmica familiar influencia de maneira negativa a qualidade de vida dos idosos dependentes, uma vez que, quanto mais prejudicada a funcionalidade familiar, pior a qualidade de vida desses.

\section{ABSTRACT}

Objective: The aim of this study was to determine the influence of sociodemographic factors, health status, functional capacity and family dynamics on the quality of life of dependent elderly individuals living at home in a city in the hinterland of Northeast Brazil. Methods: This is an analytical study with a cross-sectional design. The sample was composed of 117 dependent elderly individuals, enrolled in the Family Health Units in the district of Jequiezinho in the city of Jequié, located in the hinterland of Bahia state. The data collection instruments used were the Family Apgar, WHOQOL-OLD, Barthel's Index as well as socioeconomic and health status data. Results: The chi-square $\left(x^{2}\right)$ test showed a statistical difference between compromised quality of life and family dynamics, with the exception of the autonomy domain $(p=0.061)$ of quality of life. Conclusions: It is evident that only compromised family dynamics has a negative influence on the quality of life of the functionally dependent elderly, given that the more compromised the family functionality, the worse the quality of life of these individuals.

1 Universidade de São Paulo (USP), Escola de Enfermagem de Ribeirão Preto. Universidade Federal do Rio Grande do Norte (UFRN), Centro de Ciências da Saúde.

2 Universidade Federal do Rio Grande do Norte (UFRN).

3 Faculdade de Tecnologia e Ciências (FTC).

4 Universidade Federal do Rio Grande do Norte, Universidade Estadual do Sudoeste da Bahia (Uesb).

Endereço para correspondência: Luciana Araújo dos Reis

Rua i, 15, Urbis III, Jequiezinho - 45200.000 - Jequié, BA

Telefone: (73) 3526-7189

E-mail: cianareis@hotmail.com 


\section{INTRODUÇÃO}

Diante da realidade inquestionável das transformações demográficas iniciadas no último século, em que se observa uma população cada vez mais envelhecida, evidencia-se a importância de garantir aos idosos não só uma sobrevida maior, mas também uma boa qualidade de vida (QV)'.

A QV na terceira idade tem sido motivo de amplas discussões em todo o mundo, pois existe atualmente uma grande preocupação em preservar a saúde e o bem-estar global dessa parcela da população para que tenham um envelhecer com dignidade. A definição de QV é bastante complexa e envolve dimensões como bem-estar físico, familiar e emocional, habilidade funcional, espiritualidade, função social, sexualidade e função ocupacional, que quando integrados mantêm o indivíduo em equilíbrio consigo mesmo e com o mundo ao seu redor².

O conceito de QV está relacionado à autoestima e ao bem-estar pessoal e abrange uma série de aspectos, como a capacidade funcional, o nível socioeconômico, o estado emocional, a interação social, a atividade intelectual, o autocuidado, o suporte familiar, o próprio estado de saúde, os valores culturais, éticos e a religiosidade, o estilo de vida, a satisfação com o emprego e/ou com as atividades diárias e o ambiente em que se vive. Portanto, a definição de QV varia de autor para autor e, além disso, é um conceito subjetivo, dependente do nível sociocultural, da faixa etária e das aspirações pessoais do indivíduo³.

A QV boa ou excelente é aquela que oferece um mínimo de condições para que os indivíduos possam desenvolver o máximo de suas potencialidades, vivendo, sentindo ou amando, trabalhando, produzindo bens ou serviços; fazendo ciência ou artes, vivendo apenas enfeitando, ou, simplesmente existindo. Por outro lado, muitas pessoas procuram associar QV ao fator saúde 4 .

Assim como é difícil conceituar QV, também o é a sua medida, já que pode sofrer influência de valores culturais, éticos e religiosos, bem como de valores e percepções pessoais. Esse caráter de subjetividade de que se reveste o conceito de QV baseia-se na realidade individual que converge no subjetivismo, constituindo-se, assim, num conceito muito complexo e de difícil avaliação5.

Avaliar a QV do idoso implica a adoção de múltiplos critérios de naturezas biológica, psicológica e sociocultural, pois vários elementos são apontados como determinantes ou indicadores de bem-estar na velhice: longevidade, saúde biológica, saúde mental, satisfação, controle cognitivo, competência social, produtividade, atividade, eficácia cognitiva, status social, renda, continuidade de papéis familiares, ocupacionais e continuidade de relações informais com amigos $^{6}$.
Ante o exposto, avaliar a influência das condições sociodemográficas, de saúde, capacidade funcional e dinâmica familiar na QV de idosos dependentes reveste-se de grande importância científica e social por permitir a implementação de alternativas válidas de intervenção, tanto em programas gerontogeriátricos, quanto em políticas sociais gerais, no intuito de promover o bem-estar das pessoas maduras, particularmente, em nosso contexto, no qual os atuais idosos são aqueles que conseguiram sobreviver às condições adversas.

Essas situações determinam as implicações com relação às potencialidades de saúde e de vida do idoso, interferindo no seu processo saúde-doença. Sob essa perspectiva, este estudo tem por objetivo avaliar a influência de fatores sociodemográficos, condições de saúde, capacidade funcional e dinâmica familiar na QV de idosos dependentes residentes em domicílio na região do Nordeste

\section{MÉTODOS}

Trata-se de uma pesquisa de caráter analítica com delineamento transversal.

Os locais de estudo foram as residências dos idosos cadastrados nas Unidades de Saúde da Família da área de abrangência do bairro do Jequiezinho, no município de Jequié, situado no interior do estado da Bahia, região Nordeste do Brasil.

A amostra deste estudo foi composta por 117 idosos dependentes, cadastrados nas quatro Unidades de Saúde da Família (Padre Hilário, José Maximiliano Henríquez Sandoval, Giserlando Biondi e Júlia Magalhães) do bairro do Jequiezinho, município de Jequié, BA. O cálculo da amostra foi feito com base no Teste Piloto deste estudo, o qual foi realizado com 27 idosos participantes de um Grupo de Convivência do bairro Jequiezinho, no município de Jequié, BA.

Foram adotados os seguintes critérios de inclusão:

- Idade mínima de 60 anos, atendendo aos critérios da Política Nacional do Idoso, que considera idosa a pessoa a partir de 60 anos, e idade máxima de 80 anos;

- Em condição mental para responder aos questionários (a avaliação do estado mental do idoso foi realizada de acordo com a percepção do cuidador sobre a condição mental do idoso para participar da entrevista);

- Classificados como dependentes em suas atividades da vida diária de acordo com o Índice de Barthel’;

- Que concordassem em participar da pesquisa, firmando o Termo de Consentimento Livre e Esclarecido, segundo a Resolução 196/96 do Conselho Nacional de Saúde.

- Residir com a família.

O instrumento de pesquisa foi constituído de quatro partes. A primeira parte foi representada por dados so- 
ciodemográficos (idade, sexo, renda familiar, estado civil e escolaridade) e de saúde (presença de patologias e o uso de medicamentos). A segunda parte referiu-se ao Índice de Barthel', utilizado para avaliação funcional, composto por 10 atividades: alimentação, banho, higiene pessoal, vestir-se, intestinos, bexiga, transferência para higiene íntima, transferência-cadeira e cama, deambulação e subir escadas.

A terceira parte do instrumento foi constituída pelo Apgar de Família ${ }^{8}$, representa um acrônimo - palavra formada pela primeira letra de cada item - derivada de: "adaptation" (adaptação), "partnership" (companheirismo), "growth" (desenvolvimento), "affection" (afetividade) e resolve (capacidade resolutiva). Os domínios citados são avaliados por meio de cinco questões simples às quais são atribuídos valores, que ao final são somados, resultando num escore total cuja representação numérica relaciona-se diretamente a uma condição de funcionalidade familiar (boa funcionalidade 0 a 4, moderada - 5 e 6 ou alta disfuncionalidade - 7 a 10).

A quarta parte do instrumento foi representada pelo Questionário genérico WHOQOL-OLD9, desenvolvido pelo grupo de QV da Organização Mundial de Saúde (OMS), e que tem por finalidade medir a percepção dos indivíduos a respeito do impacto que as doenças causam em sua vida. Este Questionário consta de 24 itens, com resposta por escala tipo Likert de 1 a 5, divididos em seis facetas. Cada faceta é composta por quatro itens, gerando, então, escores que variam de 4 a 20 pontos. Os escores das seis facetas, combinados com as respostas aos 24 itens, geram, também, um escore overall (total). As facetas são: funcionamento dos sentidos; autonomia; atividades passadas, presentes e futuras; participação social; morte e morrer; e intimidade. Como cada faceta é composta por quatro itens, os escores podem variar de 4 a 20 para cada faceta. A obtenção do escore total deriva da soma dos 24 itens e não depende do agrupamento por facetas.

O WHOQOL-OLD é graduado inicialmente entre 4 e 20, sendo em seguida realizada uma nova transformação dos escores numa escala de 0 a 100, na qual o zero (0) corresponde a um pior estado de saúde e cem (100) a um melhor estado, possibilitando a análise individual de cada dimensão.

Os procedimentos de coleta de informações foram instituídos após aprovação pelo Comitê de Ética da Universidade Estadual do Sudoeste da Bahia - UESB (Protocolo no 005/2008), obedecendo à Resolução196/96 que trata das pesquisas realizadas em seres humanos. Para participar da pesquisa voluntariamente, o idoso ou seu responsável assinaram um Termo de Consentimento Livre e Esclarecido.

Os dados coletados foram organizados em banco de dados eletrônicos, por meio de digitação em planilha do Programa Estatístico SPSS versão 13.0, sendo posteriormente realizada análise estatística descritiva (média, desviopadrão, mediana, frequência e porcentagem) e aplicação do Teste do qui-quadrado $\left(x^{2}\right)$, com nível de significância de
5\%. As categorias dos domínios da QV foram criadas a partir do valor encontrado na mediana.

\section{RESULTADOS}

Foram estudados 117 idosos com idade mínima de 60 anos e máxima de 106 anos, com média de 80,79 $( \pm 9,77)$ anos. Verificou-se uma maior frequência de idosos do sexo feminino (70,09\%), não alfabetizados (63,25\%) e viúvos (46,15\%).

Os problemas de saúde estiveram presentes em 93,16\% dos idosos, enquanto $84,60 \%$ dos idosos possuem até duas patologias. As patologias mais frequentes foram a hipertensão arterial (23,10\%), acidente vascular encefálico (11,10\%) e artrose em joelhos (6,80\%). A maioria dos idosos (78,60\%) faz uso de medicação controlada, sendo o anti-hipertensivo a medicação mais utilizada (47,80\%); 81,20\% fazem uso de até duas medicações diárias, sendo mais frequente a administração duas vezes ao dia (30,80\%) e uma vez ao dia (25,60\%).

Em relação à renda familiar, 53,85\% das famílias possuem renda de 1 a 3 salários mínimos; 44,4\% dos idosos são responsáveis pelo sustento familiar e $79,5 \%$ da renda desses idosos é proveniente de aposentadoria.

Quanto à composição familiar, houve uma maior distribuição de idosos que residem com os filhos (15,40\%), com o cônjuge e filhos (12,80\%) e com filhos e netos (12,00\%). Em relação ao número de pessoas que residem com o idoso, o número mínimo foi um, o máximo quatro e a média 1,78 $( \pm 0,82)$ pessoas; $34,2 \%$ dos idosos residem com pelo menos 2 pessoas e 6,8\% residem sozinhos.

Constatou-se, quanto à funcionalidade familiar, que 73,50\% dos idosos relataram comprometimento da dinâmica familiar, sendo mais frequente a definição da dinâmica familiar como moderada funcionalidade (46,15\%).

Em relação à capacidade funcional, a média foi de 53,89 $( \pm 24,46)$ pontos, valor mínimo de zero ponto, máximo de 95 pontos e mediana de 60 pontos. Houve uma maior distribuição de idosos apresentando 60 pontos (59,00\%). Quanto à classificação do nível de dependência 53, 85\% dos idosos foram denominados como dependentes leves seguidos de 23,08\% classificados como dependentes moderados.

Observou-se que a QV se encontra mais comprometida nos domínios participação social (32,03 pontos) e funcionamento sensório (40,76 pontos) e que os idosos apresentaram melhor pontuação nos domínios morte e morrer (72,16\%) e intimidade $(53,16 \%)$ do WHOQOL-OLD, ou seja, menor comprometimento da QV.

Com a aplicação do Teste do qui-quadrado $\left(x^{2}\right)$ entre QV e as variáveis do estudo (dados sociodemográficos e condições de saúde, dinâmica familiar e capacidade funcional), verificouse diferença estatística apenas entre comprometimento da QV e os domínios da dinâmica familiar, com exceção do domínio autonomia $(p=0,061)$. 
Tabela 1. Distribuição das variáveis sociodemográficas e de saúde de idosos dependentes residentes no interior da região Nordeste. Jequié, BA, 2008

\begin{tabular}{lcc}
\hline Variáveis do estudo & N & $\%$ \\
\hline Sexo & & \\
$\quad$ Feminino & 82 & 70,09 \\
$\quad$ Masculino & 35 & 29,91 \\
$\quad$ Estado civil & & \\
$\quad$ Casado (a)/Amasiado (a) & 40 & 34,19 \\
$\quad$ Solteiro (a) & 16 & 13,68 \\
$\quad$ Viúvo (a) & 54 & 46,15 \\
$\quad$ Separado (a)/desquitado (a) & 7 & 5,98 \\
Escolaridade & & \\
$\quad$ Sim & 43 & 36,75 \\
$\quad$ Não & 74 & 63,25 \\
Problemas de saúde & & \\
$\quad$ Sim & 109 & 93,16 \\
$\quad$ Não & 8 & 6,84 \\
Tipo de medicamento 2 & & \\
$\quad$ Até 2 medicamentos & 95 & 81,20 \\
$\quad$ Acima de 2 medicamentos & 22 & 18,80 \\
Total & 117 & 100,0 \\
\hline
\end{tabular}

Fonte: Dados coletados pelos autores.

Tabela 2. Distribuição dos idosos quanto à funcionalidade familiar. Jequié, BA, 2008

\begin{tabular}{lcc}
\hline Dinâmica familiar & N & $\%$ \\
\hline 0 a 4 (boa funcionalidade) & 31 & 26,50 \\
5 e 6 (moderada funcionalidade) & 54 & 46,15 \\
7 a 10 (alta disfuncionalidade) & 32 & 27,35 \\
Total & 117 & 100,00 \\
\hline
\end{tabular}

Fonte: Dados coletados pelos autores.

Tabela 3. Distribuição dos idosos quanto à classificação segundo o Índice de Barthel. Jequié, BA, 2008

\begin{tabular}{lcc}
\hline Capacidade funcional & N & $\%$ \\
\hline$<20$ dependência total & 15 & 12,82 \\
$20-35$ dependência grave & 12 & 10,26 \\
$40-55$ dependência moderada & 27 & 23,08 \\
$\leq 60$ dependência leve & 63 & 53,85 \\
Total & 117 & 100,00 \\
\hline
\end{tabular}

Fonte: Dados coletados pelos autores.

Tabela 4. Distribuição dos domínios do WHOQOL-OLD. Jequié, BA, 2008

\begin{tabular}{lcccc}
\hline Domínios do WHOQ0L-OLD & N & Média & $\begin{array}{c}\text { Desvio- } \\
\text { padrão }\end{array}$ & (IC95\%) \\
\hline Funcionamento do sensório & 117 & 40,76 & 26,89 & 35,83 \\
Autonomia & 117 & 45,52 & 28,75 & 40,26 \\
Atividades passadas, presentes e futuras & 117 & 44,68 & 25,91 & 39,94 \\
Participação social & 117 & 32,03 & 27,49 & 24,77 \\
Morte e morrer & 117 & 72,16 & 65,88 & 65,88 \\
Intimidade & 117 & 53,16 & 26,07 & 48,39 \\
\hline
\end{tabular}

Fonte: Dados coletados pelos autores.
Tabela 5. Distribuição dos idosos quanto aos domínios do WHOQOL-OLD segundo a dinâmica familiar. Jequié, BA, 2008

\begin{tabular}{|c|c|c|c|c|c|c|c|}
\hline & \multicolumn{6}{|c|}{ Dinâmica familiar } & \multirow{3}{*}{$p$} \\
\hline & \multicolumn{2}{|c|}{$\begin{array}{c}\text { Com } \\
\text { comprometimento } \\
\end{array}$} & \multicolumn{2}{|c|}{$\begin{array}{c}\text { Sem } \\
\text { comprometimento } \\
\end{array}$} & \multicolumn{2}{|c|}{ Total } & \\
\hline & N & $\%$ & N & $\%$ & N & $\%$ & \\
\hline \multicolumn{8}{|c|}{ Funcionamento do sensório } \\
\hline$\leq 38$ & 51 & 85,00 & 9 & 15,00 & 60 & 100 & \multirow{2}{*}{0,002} \\
\hline$>38$ & 34 & 59,65 & 23 & 40,35 & 57 & 100 & \\
\hline \multicolumn{8}{|l|}{ Autonomia } \\
\hline$\leq 50$ & 51 & 79,69 & 13 & 20,31 & 64 & 100 & \multirow{2}{*}{0,061} \\
\hline$>50$ & 34 & 64,15 & 19 & 35,85 & 53 & 100 & \\
\hline \multicolumn{8}{|c|}{$\begin{array}{l}\text { Atividades passadas, } \\
\text { presentes e futuras }\end{array}$} \\
\hline$\leq 50$ & 65 & 84,42 & 12 & 15,58 & 77 & 100 & \multirow{2}{*}{0,000} \\
\hline$>50$ & 20 & 50,00 & 20 & 50,00 & 40 & 100 & \\
\hline \multicolumn{8}{|c|}{ Participação social } \\
\hline$\leq 25$ & 58 & 85,29 & 10 & 14,71 & 68 & 100 & \multirow{2}{*}{0,000} \\
\hline$>25$ & 27 & 55,10 & 22 & 44,90 & 49 & 100 & \\
\hline \multicolumn{8}{|c|}{ Morte e morrer } \\
\hline$\leq 82$ & 38 & 64,41 & 21 & 35,59 & 59 & 100 & \multirow{2}{*}{0,044} \\
\hline$>82$ & 47 & 81,03 & 11 & 18,97 & 58 & 100 & \\
\hline \multicolumn{8}{|l|}{ Intimidade } \\
\hline$\leq 57$ & 56 & 87,50 & 8 & 12,50 & 64 & 100 & \multirow{2}{*}{0,000} \\
\hline$>57$ & 29 & 54,72 & 24 & 45,28 & 53 & 100 & \\
\hline
\end{tabular}

Fonte: Dados coletados pelos autores.

\section{DISCUSSÃO}

Fatores como idade avançada, sexo, baixo nível de escolaridade e não ter companheiro estão relacionados a baixos níveis de $\mathrm{QV}^{10}$. Assim, no presente estudo, optou-se por analisar as variáveis sociodemográficas sexo, idade, escolaridade, estado civil e renda. A renda foi incluída por ser considerada também um importante indicador socioeconômico. No entanto, com a aplicação do Teste do qui-quadrado $\left(x^{2}\right)$, entre as variáveis sociodemográficas e comprometimento da QV, não foi encontrada diferença estatística.

A distribuição entre as faixas etárias está de acordo com a realidade nacional, no entanto a distribuição quanto ao sexo não reflete tal realidade, visto que a mulher figura em maior número na faixa etária acima de 60 anos $^{11}$. A predominância de idosos pertencentes ao sexo feminino tem sido atribuída à menor exposição a determinados fatores de risco, notadamente no trabalho, menor prevalência de tabagismo e uso de álcool, diferença quanto à atitude em relação a doenças e incapacidades e, por último, uma maior cobertura de assistência gineco-obstétrica ${ }^{12}$.

Quanto ao índice de escolaridade, 63,25\% dos idosos são analfabetos; esses dados são corroborados por uma pesquisa realizada no Nordeste do Brasil, no qual se constataram taxas elevadas de analfabetismo entre os idosos; aproximadamente 65 em cada 100 idosos não sabiam ler e escrever ${ }^{13}$. 
Com relação ao estado civil, os viúvos predominaram nessa amostra, com 46,15\%, seguido pelos casados, com $34,19 \%$. Considerando-se que as mulheres idosas constituem o maior número de participantes desse estudo, justifica-se o fato da menor longevidade dos homens, mas, também, da maior frequência de novo casamento dos homens, após a viuvez bem como sua maior tendência a se casarem com mulheres mais jovens ${ }^{14}$.

Em relação à renda familiar, 53,85\% das famílias possuem renda de um a três salários mínimos. De acordo com os dados da Pesquisa Nacional por Amostra de Domicílios (PNAD), cerca de 42,1\% e 40,9\% da renda familiar, nas regiões Nordeste e Sudeste, respectivamente, eram provenientes das aposentadorias ${ }^{15}$. Uma boa situação socioeconômica mostra-se associada à melhor QV.

Percebe-se que nas alterações relacionadas à idade estão a presença de fatores de risco e a ocorrência de doenças crônicodegenerativas, que determinam para o idoso certo grau de dependência, diretamente relacionada a perda de autonomia e dificuldade de realizar as atividades básicas da vida diária, interferindo na sua QV'16.

A capacidade de o indivíduo realizar suas atividades físicas e mentais, necessárias para a manutenção de suas atividades básicas e instrumentais, ou seja, tomar banho, vestir-se, realizar higiene pessoal, transferir-se, manter a continência, preparar refeições, controle financeiro, tomar remédios, arrumar a casa, fazer compras, usar transporte coletivo, usar telefone e caminhar certa distância, é definida como capacidade funcional ${ }^{17}$.

A manutenção e a preservação da capacidade para desempenhar as atividades básicas de vida diária são pontos básicos para prolongar o maior tempo possível a independência; com isso, os idosos mantêm sua capacidade funcional e QV ${ }^{7}$. A conservação da capacidade funcional pode ter importantes implicações na QV dos idosos, por estar relacionada à capacidade de se ocupar em desenvolver atividades cotidianas e/ou atividades agradáveis ${ }^{18}$. No entanto, na presente pesquisa, não foi encontrada diferença estatística entre comprometimento da capacidade funcional e QV.

Diante das transformações nas estruturas familiares, questiona-se em que medida as modificações causadas pela presença de idosos, com limitações importantes na dinâmica de funcionamento das famílias, bem como as consequências disso no equilíbrio da estrutura familiar, passam a estar cada vez mais presentes. Supõe-se que, em condições de disfuncionalidade, as famílias possam ter a sua capacidade assistencial prejudicada e, assim, não consigam prover adequadamente $\mathrm{o}$ atendimento sistemático das necessidades de cuidados a seus parentes idosos, podendo dessa forma interferir na QV dos idosos ${ }^{19}$.

No presente estudo, constatou-se que o comprometimento da dinâmica familiar influencia de maneira negativa a QV dos idosos dependentes, uma vez que quanto mais comprometida a funcionalidade familiar, mais prejudicada é a QV desses idosos $20-21$.

Os idosos com comprometimento da dinâmica familiar apresentam prejuízo do domínio referente ao funcionamento do sensório no WHOQOL-OLD. Isso pode ser justificado pelo fato de que, por esse domínio corresponder a perda dos sentidos (audição, visão, paladar, olfato e tato) que acabam por afetar a vida diária, a capacidade de participar em atividades e a capacidade de interagir com outras pessoas, tornam o idoso dependente dos cuidados da família. O suporte familiar contribui de maneira significativa para a manutenção e a integridade física e psicológica do indivíduo. Seu efeito é tido como benéfico, no membro da família que o recebe, na medida em que o suporte é percebido como disponível e satisfatório22.

O domínio do WHOQOL-OLD referente à autonomia, o qual é representado pelos itens liberdade de tomar decisões, controlar o próprio futuro e ter a sua autonomia respeitada pelas pessoas que o cercam, foi o único que não apresentou diferença estatística, quando associado à dinâmica familiar.

Outro domínio afetado negativamente pelo comprometimento da dinâmica familiar foi constituído por atividades passadas, presentes e futuras, em que os idosos relataram não estar satisfeitos com a possibilidade de continuar alcançando realizações pessoais e satisfação com o reconhecimento pela família e com o que alcançou em sua vida. Dessa forma, compreende-se ser fundamental para o planejamento assistencial adequado ao idoso a compreensão de seu contexto familiar, o que implica a compreensão das questões que envolvem a formação e a dinâmica de funcionamento das famílias em geral.

Em relação ao domínio participação social da QV, que se refere ao uso do tempo livre e a participação em atividades da comunidade pelos idosos, esse também apresentou diferença estatística com o comprometimento da dinâmica familiar, assim como o domínio intimidade, em que são avaliadas questões relativas a companheirismo, capacidade de amar e se amado. As pessoas idosas, que estão bem integradas às suas famílias e ao seu meio social, têm maiores chances de sobrevivência, além de concentrar melhor capacidade de se recuperar das doenças, sendo o isolamento social importante fator de risco para a morbidade e mortalidade. A saúde de cada membro familiar afeta o funcionamento da família da mesma forma que o funcionamento desta afeta a cada um dos membros ${ }^{24}$.

O domínio morte e morrer refere-se ao grau de preocupação do idoso com a maneira em que irá morrer, medo de não poder controlar a sua morte, medo de morrer e sentir dor antes de morrer, e também apresentou diferença estatística quando relacionado ao comprometimento da dinâmica familiar. Percebe-se que, nas questões referentes a esse domínio, estão associadas questões de 
espiritualidade, uma vez que boa parte dos idosos entrevistados acredita que a morte é a única coisa certa que temos na vida, sendo assim, não devemos temê-la e sim aceitá-la com humildade.

Portanto, observa-se a necessidade cada vez mais premente de serem estabelecidos esquemas assistenciais mais efetivos e dinâmicos, capazes de atender às demandas crescentes dos idosos e de suas famílias, de forma a permitir que ambos encontrem, conjuntamente, uma solução terapêutica mais efetiva e adequada em que o equilíbrio familiar seja a meta, melhorando assim a assistência aos idosos e diminuindo os custos emocionais da própria família, favorecendo a uma melhora na QV desses idosos.

\section{REFERÊNCIAS}

1. Reis LA, Torres GV, Silva JPA, Sampaio LS, Reis LA. Perfil epidemiológico de idosos institucionalizados no Município de Jequié/BA. Rev Enfermagem Atual. 2008:46:19-23.

2. Heinonen $H, A r o A R$, Aalto AM, Utela A. Is the evaluation of the global quality of life determined by emotional status? Qual Life Res. 2004;13(8):1347-56.

3. Sousa L, Galante H, Figueiredo D. Qualidade de vidas e bem-estar dos idosos: um estudo exploratório na população portuguesa. Rev Saúde Pública. 2003;37(3):364.

4. Garcia EL, Banegas JR, Perez-Regadera AG, Cabrera RH, Rodriguez-Artalejo F. Social network and health related quality of line in older adults: a population-based a study in Spain. Qual Life Res. 2005;14(2):511-20.

5. Thomé B, Dykes AK. Hallberg IR. Quality of life in old people with and without cancer. Qual Life Res. 2004;13(6):1067-80.

6. Chikude T, Fujjki EM, Honda EK, Ono NK, Milani C. Avaliação da qualidade de vida dos pacientes idosos com fratura de colo de fêmur tratados cirurgicamente pela artroplastia parcial do quadril. Acta Ortop Bras. 2007;15(4):32-40.

7. Reis LA, Mascarenhas CHM, Torres GV. Evaluation of functional capacity on institutionalized elderly in the City of Jequié/BA. Fiep Bulletin. 2008;78(1):89-92.

8. Mazza MMPR, Lefevre F. Cuidar em família: análise da representação social de relação do cuidador familiar com o idoso. Rev Bras Crescimento Desenvolv Hum. 2005;15(1):1-10.

9. Fleck MP, Chachamovich E, Trentini CM. Projeto WHOQOL-OLD: método e resultados de grupos focais no Brasil. Rev Saúde Pública. 2003:37(6):793-99.
10. Carneiro RS, Falcone E, Clark C, Del Prette Z, Del Prette A. Qualidade de vida, apoio social e depressão em idosos: relação com habilidades sociais. Psicol Reflex Crít. 2007;20(2):27-35.

11. Reis LA, Mascarenhas CHM, Marinho Filho LE, Borges OS. Lombalgia na terceira idade: distribuição e prevalência na Clínica Escola de Fisioterapia da Universidade Estadual do Sudoeste da Bahia. Rev Bras Geriatr Gerontol. 2008;11(1):93-103.

12. Reis LA, Mascarenhas CHM, Costa AN. Lessa RS. Estudo das condições de saúde de idosos em tratamento no setor de neurogeriatria da Clínica Escola de Fisioterapia da Universidade Estadual do Sudoeste da Bahia. Rev Baiana Saúde Pública. 2007;31(2):324-32.

13. Garcia RA, Carvalho JAM. 0 envelhecimento da população brasileira: um enfoque demográfico. Cad Saúde Pública. 2003;19(3):725-33.

14. Davim RMB, Torres GV, Dantas SMM, Lima VM. Estudo com idosos de instituições asilares no município de Natal/RN: características socioeconômicas e de saúde. Rev Latino-Am Enfermagem. 2004;12(3):518-24.

15. Giatti L, Barreto SM, Lima MFC. Condições de saúde, capacidade funcional, uso de serviços de saúde e gastos com medicamentos da população idosa brasileira: um estudo descritivo baseado na pesquisa nacional por amostra de domicílios. Cad. Saúde Pública. 2003;19(3):735-43

16. Parayba MI, Veras R, Melzer D. Incapacidade funcional entre as mulheres idosas no Brasil. Rev Saúde Pública. 2005;39(3):53-9.

17. Duayer MFF, Oliveira MMC, Gaspar JC. Perfil dos pacientes com perdas funcionais e dependência atendidos pelo PSF no município de São Paulo. Rev Esc Enferm USP. 2007:41(4):42-9.

18. Guimarães LHCT, Galdino DCA, Martins FLMM, Abreu SR, Lima M, Vitoriano DFM. Avaliação da capacidade funcional de idosos em tratamento fisioterapêutico. Rev Neurociências. 2004;12(3):22-9.

19. Trentini CM, Chachamovich E, Figueiredo M, Hirakata VN, Fleck MPA. A percepşão da qualidade de vida do idoso avaliado por si próprio e pelo cuidador. Estudos de Psicologia (Natal). 2006;11(2):43-52.

20. Pereira RJ, Cotta RMM, Franceschini SCC, Ribeiro RCL, Sampaio RF, Priore SE, et al. Contribuição dos domínios físico, social, psicológico e ambiental para a qualidade de vida global de idosos. Rev Psiquiatr. 2006; 28(1):27-38.

21. Abreu NS, Baracho ES, Tirado MGA, Dias RC. Qualidade de vida na perspectiva de idosas com incontinência urinária. Rev Bras Fisioter. 2007:11(6):46-51.

22. Subasi $F$, Hayran 0. Evaluation of life satisfaction index of the elderley people living in nursing homes. Arch Gerontol Geriatric. 2005;41:23-9.

23. Arnold R, Ranchor AV, Sanderman R, Kempen GI, Ormel J, Surmeijer TP. The relative contribution of monais of quality of life to overall quality of life for different chronic diseases. Qual Life Res. 2004;13(5):883-96.

24. Sampaio $L S$, Lessa RS, Reis $L A$, Torres GV. Prevalência de patologias ortopédicas em idosos atendidos em hospital público no município de Lajedo do Tabocal, BA. Rev Enferm Atual. 2007; $1(41): 30-2$ 